\title{
Quality Assessment of 12 Lead ECG Signals based on Beat Detection Pattern
}

\author{
Muhammad Yazid, B. Eng., M.Eng. \\ Department of Biomedical Engineering \\ Faculty of Electrical Technology \\ Institut Teknologi Sepuluh Nopember, \\ Surabaya, Indonesia \\ Email: yazid@bme.its.ac.id
}

\begin{abstract}
Due to limitation in computing power, available memory and power supply, ECG signal acquisition system based on mobile device needs specifically designed signal processing method which can do the required task with minimal cost. This paper proposed a new method for assessing signal quality from 12 lead ECG signal for use in mobile device based ECG signal acquisition system. The proposed method can be applied to incoming ECG data stream in one pass, does not involve computatively expensive filtering and does not require large memory space, which makes it especially useful for use in mobile device based ECG signal acquisition. The proposed method is verified on PhysioNet/Computing in Cardiology Challenge 2011 12 lead ECG signals database, achieving a result of 89.98 percent accuracy when tested against the training dataset, and 87.4 percent accuracy when tested against the test data set.
\end{abstract}

Keywords-ECG signal quality assessment

\section{INTRODUCTION}

Recent years have seen increasing proliferation of mobile electronic devices such as smartphones and tablets. The advances in mass production technology reduced the cost of these devices such that it becomes easily available even for people in rural areas of developing countries. Coupled with improvement in communication network coverages and data capacities, this phenomena has opens up many insteresting opportunities for its application in improving the quality of life of people in rural communities.

In archipelagic countries such as Indonesia, limitations in funding and human resources has cut off many people who lives in remote communities from basic health care. While on one hand providing such service is the responsibility of every government toward its citizens, building specialized health care institution with expensive equipment and staffed by highly trained medical professionals in remote places with small number of resident is not feasible.

One possible solutions is to distribute low cost smartphone based health signal data aquisition system, which can be used to gather health data from patients in remote places. This data will then be transmitted through mobile data communication networks to servers in central health care institutions, where it can be further analyzed by people with specialized training using high quality equipment.
Because the distributed system is aimed to be used by people with minimal training, and with possible use in very remote places where data transmission network will not be reliable all the time, it will be crucial to have an ability to asses the quality of the signals being gathered on site, by the device it self, so that the measurement error can be immediately fixed and/or repeated when necessary.

ECG (Electro Cardio Graphy) signal measurement is an essential part of heart desease diagnosys, which is necessary to accurately determine the cause of any heart problem in a patient in order to be able to proscribe a suitable medication of other types of treatments. Given the change in human diet and livestyles, recently there is marked increase in the number of heart disease related death, which adds to the importance of providing heart disease check up service for all patients, including those in remote places.

Because standard ECG signal measuring equipment is expensive, bulky and require specialized training to operate, development of low cost mobile ECG signal acquisition would be highly beneficial for health disease prevention in rural areas of developing countries. For this, a reliable method of assessing measured ECG signal quality based on mobile devices onsite would be necessary.

The main objective of this work is to contribute to this effort by developing reliable ECG signal quality assessment method which is suitable for use in mobile devices.

\section{Previous Work on ECG Signal Quality ASSESSMENT}

Previously there have been multiple published results of attempt at developing ECG signal quality assessment method.

The work by [1] use a cascaded multiple step detector, where each steps detects specific signal defect features, starting from simple features such as flat line and saturation, to more complex features such as baseline drift low amplitude, high amplitude, and step slope. The method proposed by [1] managed to be able to have a detection accuracy of $85.7 \%$ on the test dataset. While the ability to differentiate between types of signal defects, [1] used many hardcoded threshold values in its implementation, which will make it difficult to generalize, as a different set of threshold values will have to be determined for different hardware. 
[2] developed a real-time ECG signal quality assessment method based on detection of four different signal defects: straight line, huge impulse, gaussian noise, and error in $\mathrm{R}$ wave detection. This work shares similarities with [1] in its dependence on several hard coded threshold values.

[3] used several metrics which quantify features such as spectral energy distribution, higher energy moments or interchannel agreements, and use them to train multiple machine learning algorithms. Results of multiple machine learning classification algorithms are then combined to give an added level of accuracy. Compared to [1], the work by [3] offers more flexibility for generalization, at the cost of more complicated process and computing resource requirement.

[4] and [5] presented ECG signal quality assessment methods which take advantage from the redundancy of information in a set of multiple lead ECG signals. These works generally proposed methods which try to reconstruct signal on an ECG lead from signals on other leads. By comparing the original signal from measurement its corresponding reconstructed signal, the authors can determine if there are errors in measurement which resulted in corrupted measurement results.

The method proposed by [4] is able to not only assess overall quality of ECG measurement results, but also identify specific measurement errors such as switched electrodes. In its implementation, [4] calculates the linear correlation between original ECG and reconstructed ECG using a function in Matlab, a personal computer based proprietary mathematical calculation software developed by Mathworks, Inc., which means further improvement is still necessary to be able to implement this method directly on mobile devices.

\section{Proposed ECG Signal Quality Assessment METHOD}

This paper propose a method of assessing quality of ECG signal based on anomaly in beat detection results. This method is based on the fact that most biomedical signal, including ECG, is mainly periodical. A fault in measurement, such as loose leads or imperfect connection, will result in deviation from the normal periodic signal waveform. The proposed method detect and quantify this deviation in order to assess the quality of the ECG signal.

\section{A. Beat Detection from ECG Signal}

In order to see irregular pattern in beat detection results, first we need to be able to detect heart beat the ECG signals. Because the ECG Signal Quality Assessment method is intended to be implemented in a mobile device with limited computing resources such as computing speed, memory size and battery life, the algorithm for heart beat detection has to be as simple as possible.

Since every heart beat in a normal ECG signal is always accompanied by the QRS waveform, generally detecting heart beat can be done using a QRS detection algorithm. A QRS detection algorithm based on moving window integrator by [7] is widely known to give good results and is used as a base by other works [6]. [8] improved on [7] by using patient independent adaptive threshold.
[9] leveraged the availability of multiple lead signals and proposed new algorithm which is adaptable to multiple leads and use an adaptive threshold which responds to slew rate, high frequency noise, and low amplitude beats.

The algorithm proposed by [9] first differentiated all ECG signals, and summed their absolute values into a "complex lead" signal $Y$ defined by Equation 1, where $Y(i)$ is the complex signal value at sample point $i, X_{j}(i)$ is the amplitude value of ECG lead number $j$ at sample point $i$, and $L$ is the number of leads.

$$
Y(i)=\frac{1}{L} \sum_{j=1}^{L} a b s\left(X_{j}(i+1)-X_{j}(i-1)\right)
$$

Equation 1 was adapted from an earlier work by [10], adding the conversion to absolute value and the normalizing coefficient $\frac{1}{L}$ [9].

The summing of absolute differentiated signal values in Equation 1 was deemed convenient by [9] in order to generalize their proposed algorithm to any number and kinds of ECG leads, which might have positive or negative deflection at the beat point.

The conversion of differentiated signal values into their absolute values will necessarily remove important information regarding the time when those differentiated signals crosses zero. This is especially so when the sampling period is wide (at low sampling frequency) which makes the possibility of zero values being in the middle between two differentiated signal values higher. This will result in complex signal $Y$ does not reach zero during the peaks corresponding to QRS waves, hence making it looks similar to regular (non QRS wave related) peaks, except by its amplitude.

Further, summing absolute values of the differentiated signal values means all peaks of the $Y$ signal are now in the positive. One significant features we can observe from a sum of differentiated ECG signal values is that there is a large negative peak at the position corresponding with the QRS wave. Detecting this peak as a negative value means the detector will have less noisy signal to process, as the positive peak noises can be ignored.

Using assumption that the summed differentiated ECG signals will always cross zero downward during the QRS wave, we can easily design an algorithm to only consider a negative peak in the summed differentiated signals when it happened immediately after a negative direction zero crossing.

This will significantly reduce the number of peaks that needs to be analyzed, compared to just using amplitude values as in [9].

\section{B. Simple Beat Detection for Signal Quality Assessment in 12 Lead ECG}

Since the developed algorithm is intended for use in mobile devices with limited computing resources, it must be as simple as possible. Our beat detection implementation is as follows:

1) Get summed differentiated value of ECG signals from all leads as $y$.

2) Find downward zero crossing of $y$, assign the time value of this crossing point as $t_{1}$ 


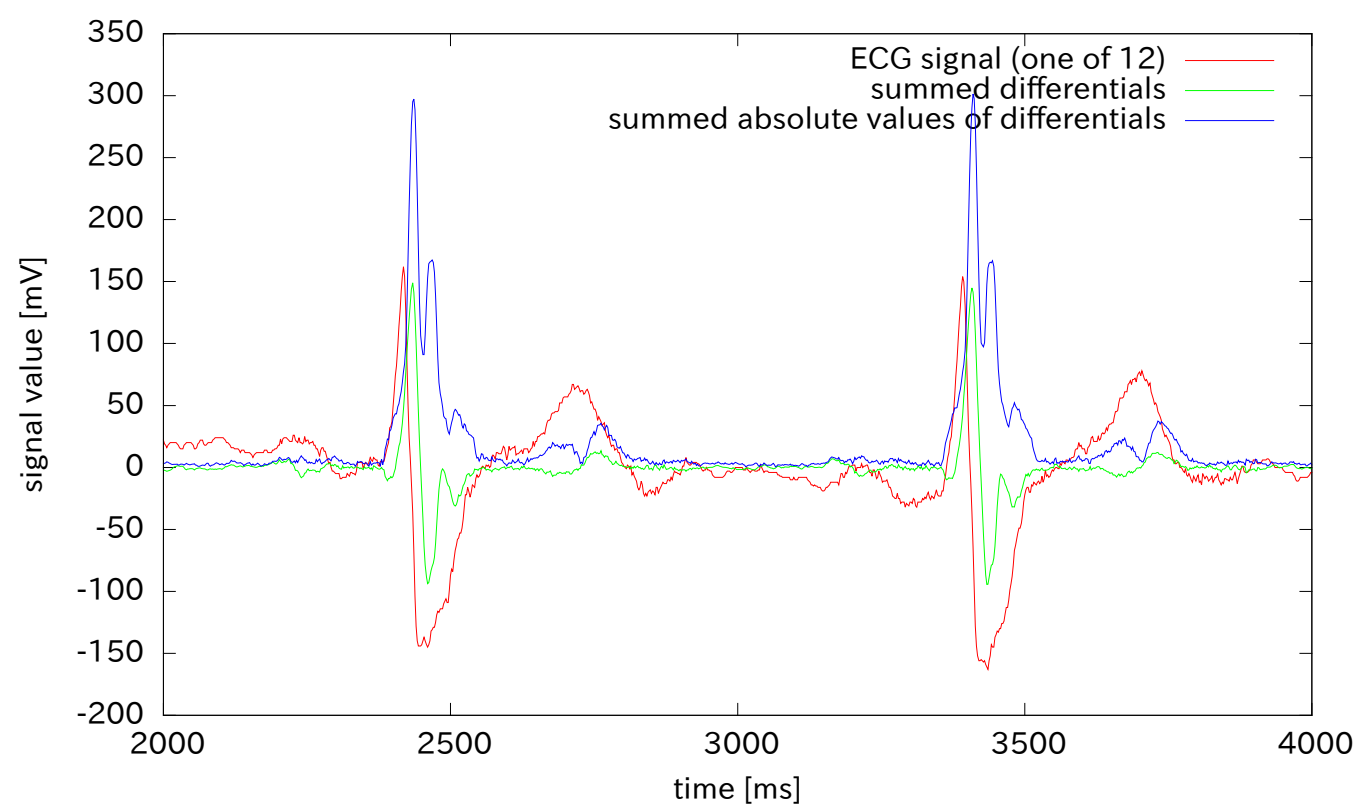

Fig. 1: Example of ecg signal (1 of 12), summed differentiated 12 ECG signals and summed absolute of differentiated 12 ECG signal. The summed values are normalized by $\frac{1}{L}$ as in Equation $1(L=12)$

3) If downward zero crossing is found, then find the minimum value of $y$ after $t_{1}$ and before any upward crossing of threshold value $v t h$. We name this minimum value $b_{0}$, and the time of its occurance as $t_{2}$. The time when $y$ crosses $v t h$ upward should be called $t_{3}$.

4) After $t_{3}$ is found, the next step is determined as follows:

a) The value of $t_{1}$ and $b_{0}$ is compared to previously found values of the same. If the current $t_{1}$ close the previous $t_{1}$ and the current $b_{0}$ is more negative than the previous one, we select the current $t_{1}$ as candidate value for the beat position and set $v t h=0.5 b_{0}$.

b) If the difference between the current $t_{1}$ and the previous $t_{1}$ is larger than a minimum delay, then we compare $b_{0}$ with $v t h$. If $b_{0}$ is larger than $v t h$, then we decide that we have found a new beat at the time $t=t_{2}$. As a special case, if there is no other beat position previously found, then the previous $t_{2}$ is also determined as a beat position. Finally, we update the value of $v$ th to $v t h=$ $0.5 b_{0}$.

5) Repeat again from step (2) until there is no more data to process.

This algorithm can be implemented as a single pass, where each step is applied to every new data point as it is received. It also has a very low memory requirement, as there is no need to record every calculated parameters for the whole length of input signal. For example, it is not necessary to save the differentiated value $y(i)$ for all $i$ from 0 to the end of the input signal.

While it is possible to increase noise tolerance of this beat detection algorithm, it is not necessary for the purpose of ECG signal quality assessment as intended in this work. The noise response of the algorithm will be useful as an indicator of signal quality.

\section{ECG Signal Quality Assessment based on Beat Detection Pattern}

Using the beat detection algorithm as described in previous section, we classify ECG signals based on its regularity and the existence of signal defect features such as noise or excessively large amplitude. A normal ECG signal will have beats with regular intervals, which is usually between $500 \mathrm{~ms}$ to $2000 \mathrm{~ms}$ (corresponding to $30 \mathrm{bpm}$ to $120 \mathrm{bpm}$ ). Any irregularity in the input ECG signals, such as flat line, noise or sudden large amplitude change, is expected to change the regularity of the beat detection result.

The Physionet Challenge in Cardiography 2011 database is a set of database consists of large number of 12 Lead ECG Signals taken with mobile ECG recording device by person with minimal training. Some of the ECG data is provided with annotation stating whether it is an "Acceptable" (good quality) ECG signals or "Unacceptable" (bad quality). This set of data is called the "training data set", or "set-a". Other ECG data set is provided without annotation, but a score representing the accuracy of our quality assessment algorithm will be provided if we upload our classification result to a remote server. This data set is called the "test data set", or "set-b". There are 1000 12 Lead ECG signals in the training data set, and 500 in the test data set.

1) Behavior of beat detection algorithm on an ordinary acceptable signal: An example of the proposed beat detection 
algorithm applied on of the "acceptable" ECG signals is shown in the Figure 2. In Figure 2, the top 12 graph each represents individual ECG leads. At the bottom left are the plot of the summed differentiated signal and the threshold value used for detecting heart beat. The bottom right graph is a plot showing each heart beat as a vertical line at its position in time. It can be seen that the beat detection algorithm successfully detect each beat, resulting in a regular beat interval.

2) Behavior of beat detection algorithm on an unacceptable signal with large noise: Figure 3 shows the beat detection algorithm applied to an ECG signal with large noise. Large noise is clearly visible in the left signal in second row from the top, and in both left and right signal in the sixth row from the top in 3. These noises are showing in the summed differentiated signals in the bottom left, and will cause a disruption on the beat detection, resulting in false detection of beats with very small intervals. While human with some heart disease might have faster heart beat, there is a minimum beat interval which can not occur naturally in a living human. By detecting and counting such unnatural beat detections it will be possible to detect an unnacceptable ECG measurement results.

3) Behavior of beat detection algorithm on a signal with sudden large amplitude change: An example of ECG signals with very large, sudden large amplitude change is shown in Figure 4

In Figure 4, unnatural sudden large amplitude changes can be seen in the left of the sixth row from the top. The very large amplitude change of this signal will translate to a very large differentiated value at that point in time, which when added to the time differetiated values of other signal, will create a very large spike. Since threshold value of the beat detection algorithm is determined by the last spike detected, the individually very large spike will result in a very large threshold value, which will not detect any subsequent "normal" spike resulted from ordinary QRS wave in other ECG signals. The beat detection result in this case will have a very large intervals (corresponding to the intervals of the sudden large amplitude changes), or will only have beats up to the point when the sudden large amplitude change occurs, as seen in Figure 5.

4) Detection of flat line signals: While flat lines in all 12 ECG leads can be easily detected by the absence of beats in beat detection results, a flat line in only some of the leads (with other leads normal) is harder to detect from the beat detection results only. One possible way to detect it without expensive additional computation is by taking the sum of the differentiated value of each signals. The beat detection algorithm already needs a differentiation of each ECG signal, so additional step required is simply summation of the differentiated value for each signal into an accumulator.

A signal with flat line (DC only) from start to end will have zero or near zero result for this sum. An example for this can be seen in Figure 6. Flat line at ECG lead no 11 (left side of sixth row from the top) corresponds to zero value in the summed differentiated value of that signal (visible in the right side of the bottom row, where the $\mathrm{x}$ axis represents ECG lead number).
Another kind of flat line signal is a signal which is only flat before or after a certain period of non flat interval. In order to be able to detect this type of condition, instead of just summing all differentiated signal value for the entire length of the signal, we reset the summation result each time a large signal change is detected, and keep the value of the longest flat period in the signal.

\section{AlgORITHM FOR QUALITY ASSESSMENT OF 12 LEAD ECG SIGNALS BASED ON BEAT DETECTION}

Based on the analysis in the previous section, we can develop the following criteria for an acceptable ECG signals using beat detection based quality assessment, each criteria applied in cascade after the previous ones:

1) If beats not detected, then the ECG signals in unacceptable.

2) If detected beat intervals are very short or very wide, then the ECG signals is unacceptable. The threshold for very short and very wide beat interval is determined based on the medically accepted natural lower and higher limit of a living human heart beat frequency, which is between $30 \mathrm{bpm}$ (beat per minute) to $100 \mathrm{bpm}$. This corresponds to shortest acceptable beat interval threshold of around $500 \mathrm{~ms}$ and widest beat interval threshold of around $2 s$.

3) If detected beats starts or ends very far from the start or end of ECG signal (i.e., there is a long interval of no beat detection at the beginning or the end of detection period), then the ECG signals in unacceptable. The threshold for the no-beat interval at the beginning and ends of ECG signals is set to the value of maximum beat intervals detected.

4) If beats detected normally, but there is one or more lead whose summed differentiated value equals zero, then the ECG signals is unacceptable.

5) Finally, if the ECG signals does not met all of the above criteria, it is considered as acceptable.

\section{RESULT}

Applied against the training data set of the "The Physionet Challenge in Cardiography 2011" database, the proposed algorithm is able to accurately detect 714 out of 773 "accepted" signals, and 182 out of 225 "unaccepted" signal, resulting in 89.78 percent overall accuracy. When applied against the test dataset and submitted to the official server for scoring, the proposed algorithm results has a score of 87.4 percent. The comparison between this work and published results can be seen in Table I.

The results in Table I shows that our proposed simple and low computing resource method can achieve similar results to previously published ECG signal quality assessment methods.

\section{CONCLUSION}

We proposed a simple ECG signal quality assessment based on beat detection. The beat detection algorithm itself is designed to be simple and requiring low computing resources, 


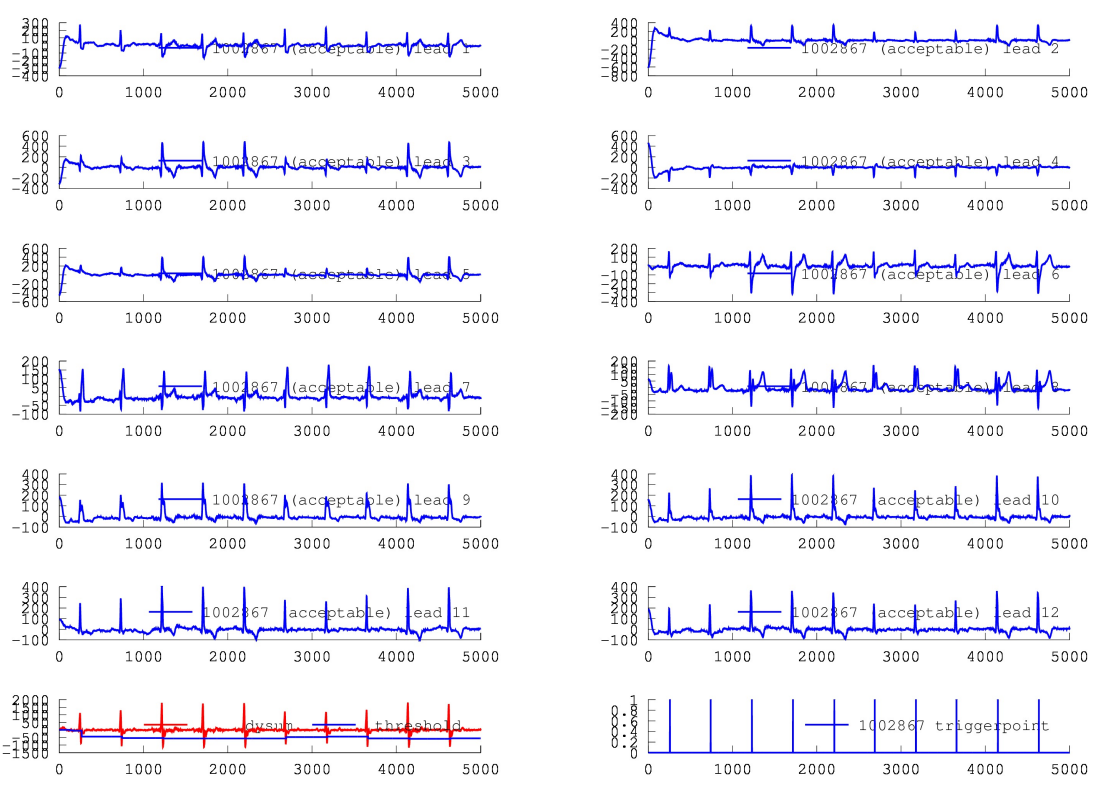

Fig. 2: Example of proposed beat detection method applied on signal number 1002867 in the "acceptable" training data set.
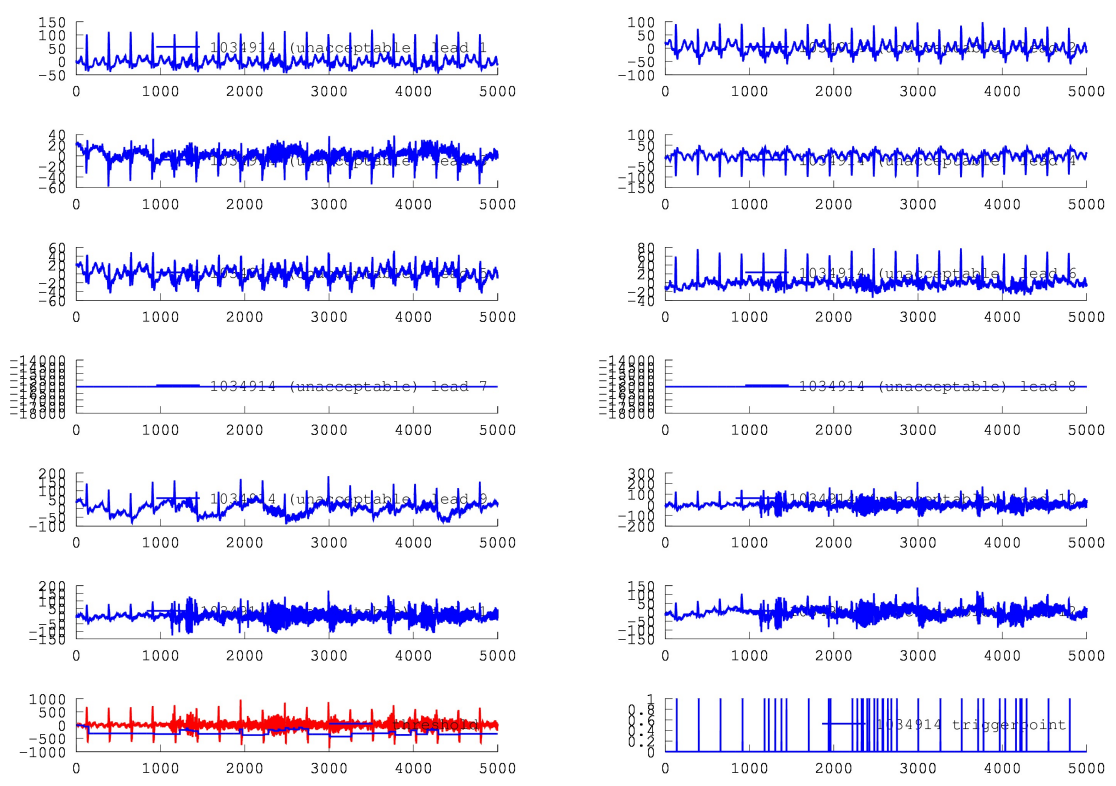

Fig. 3: Example of proposed beat detection method applied on signal number 1034914 in the "unacceptable" training data set. 

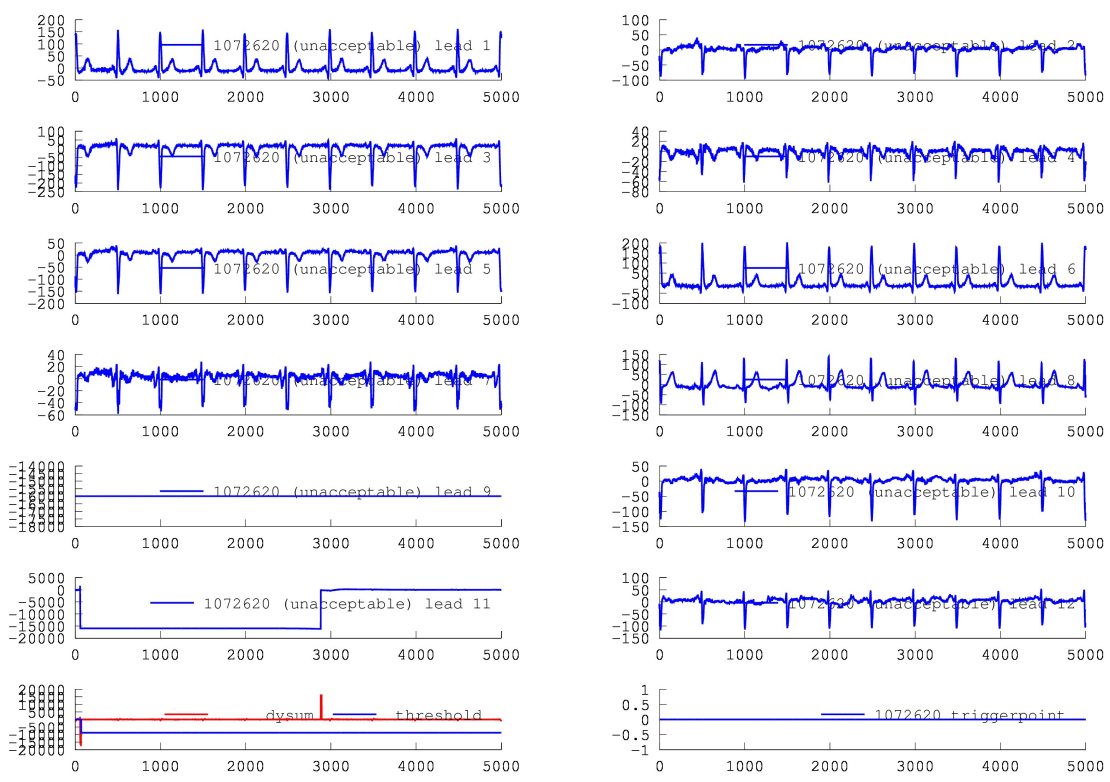

Fig. 4: Example of proposed beat detection method applied on signal number 1072620 in the "unacceptable" training data set.
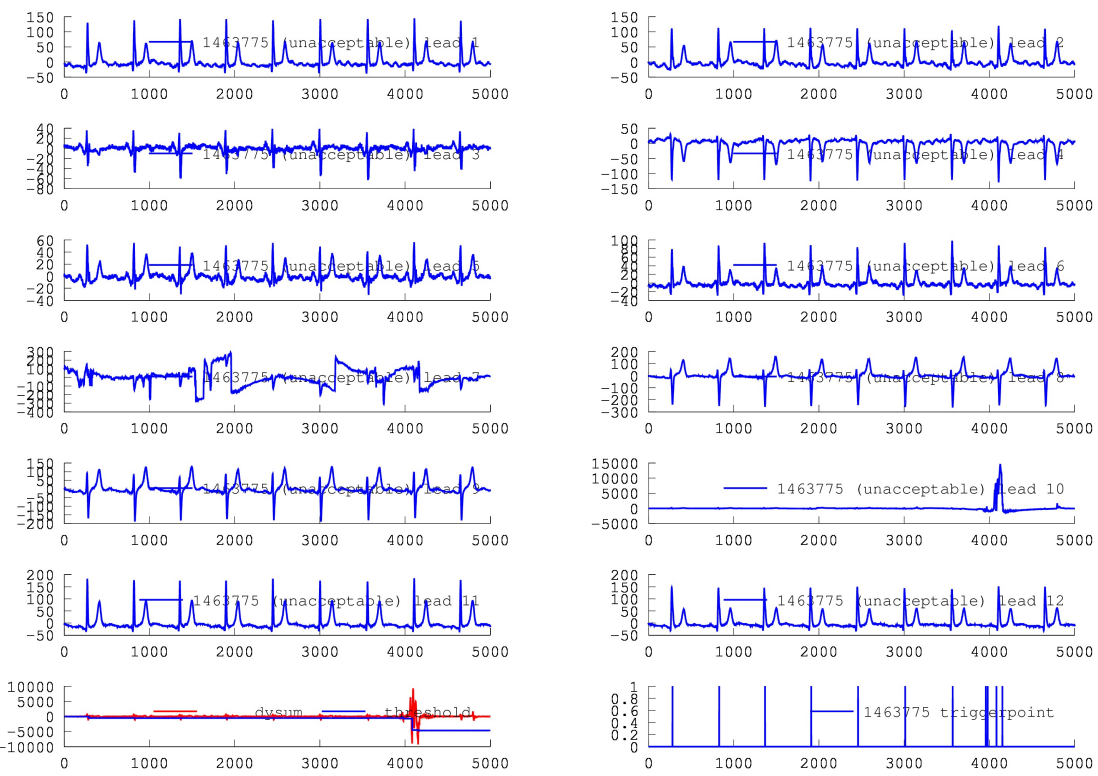

Fig. 5: Example of proposed beat detection method applied on signal number 1463775 in the "unacceptable" training data set. 

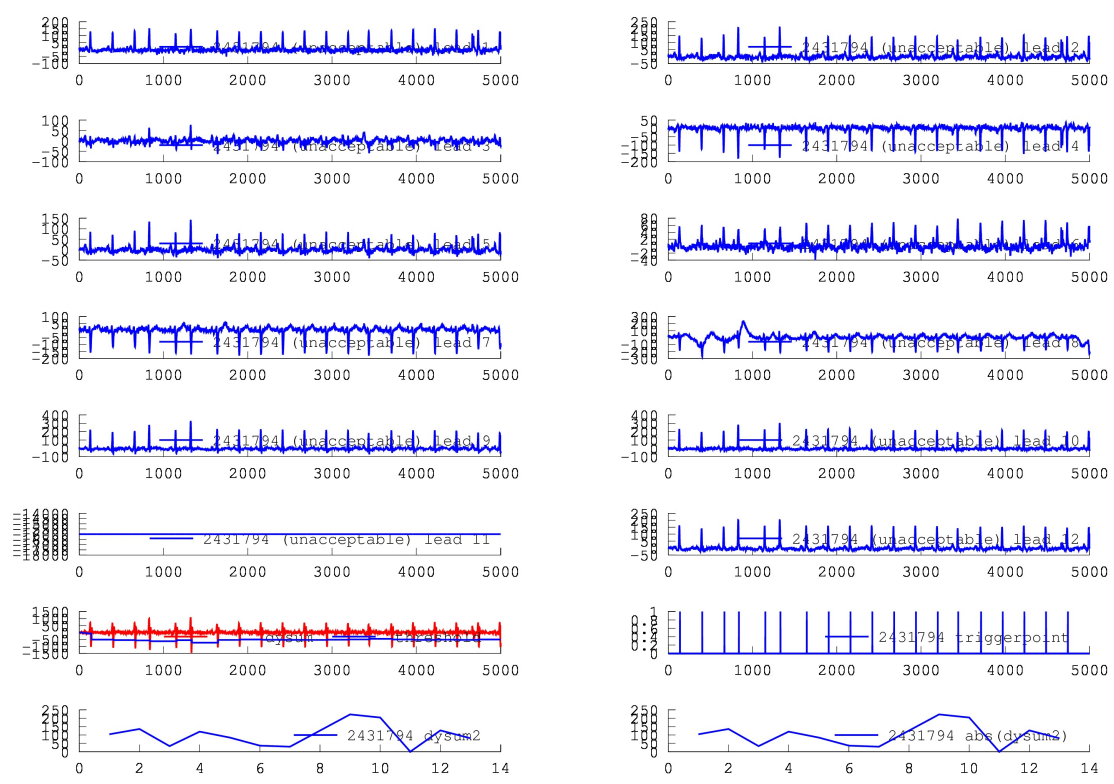

Fig. 6: Example of proposed beat detection method applied on signal number 2431794 in the "unacceptable" training data set.

TABLE I: Result Comparison Table of Proposed Method and Published Works

\begin{tabular}{|c|c|}
\hline Published by & Result Against Test Data Set (Set-B) [percent] \\
\hline Zaun, et. al. [11] & 90.4 \\
\hline Philip, et. al. [1] & 85.7 \\
\hline Clifford, et. al. [3] & 95 \\
\hline Maan, et. al. [4] & 92.2 \\
\hline Dieter, et. al. [21] & 91.6 \\
\hline Liu, et. al. [2] & 90.67 \\
\hline Moody, et. al. [12] & 89.6 \\
\hline Noponen, et. al. [5] & 90.0 \\
\hline Xia, et. al. [13] & 85.0 \\
\hline Jekova, et. al. [14] & 90.8 \\
\hline Johannesen, et. al. [15] & 88.0 \\
\hline Nir, et. al. [16] & 91.2 \\
\hline Tat, et. al. [17] & 92 \\
\hline Vito, et. al. [18] & 81.8 \\
\hline Vaclav, et. al. [19] & 82.8 \\
\hline Jakub, et. al. [20] & 83.6 \\
\hline this work & 87.4 \\
\hline
\end{tabular}

to enable the algorithm to be used in mobile devices which has limited capabilities. Our proposed algorithm is able to assess the quality of ECG signals relatively accurately, with results that is comparable to previously published methods, and in real time, which enable mobile ECG recording users to know whether the measurement needs to be repeated or wire connection fixed. Due to the low resources requirement, this algorithm will enable development of low cost mobile ECG recording device which can be distributed to low income communities in remote places, potentially improving quality of life of people living in such places.

\section{REFERENCES}

[1] P. Langley et al., "An algorithm for assessment of quality of ECGs acquired via mobile telephones," 2011 Computing in Cardiology, Hangzhou, 2011, pp. 281-284.

[2] Chengyu Liu, Peng Li, Lina Zhao, Feifei Lio, Ruxiang Wang, "Real-time Signal Quality Assessment for ECGs Collected Using Mobile Phones," 2011 Computing in Cardiology, Hangzhou, 2011, pp. 357-360.

[3] G. D. Clifford, D. Lopez, Q. Li and I. Rezek, ”Signal quality indices and data fusion for determining acceptability of electrocardiograms collected in noisy ambulatory environments," 2011 Computing in Cardiology, Hangzhou, 2011, pp. 285-288.

[4] Arie C Maan, Erik W van Zwet, SumChe Man, Suzanne M M OlivieraMartens, Martin J Schalij, Cees A Swenne, "Assessment of Signal Quality and Electrode Placement in ECGs using a Reconstruction Matrix," 2011 Computing in Cardiology, Hangzhou, 2011, pp. 289-292.

[5] Kai Noponen, Mari Karsikas, Suvi Tiinanen, Jukka Kortelainen, Heikki Huikuri, Tapop Seppanen, "Electrocardiogram QUality Classification based on Robust Best Subsets Linear Prediction Error," 2011 Computing in Cardiology, Hangzhou, 2011, pp. 353-356.

[6] lvarez, Ral Alonso, Arturo J. Mndez Penn, and X. Antn Vila Sobrino. "A comparison of three QRS detection algorithms over a public database." Procedia Technology 9 (2013): 1159-1165.

[7] Pan, Jiapu, and Willis J. Tompkins. "A real-time QRS detection algorithm." IEEE transactions on biomedical engineering 3 (1985): 230-236.

[8] Hamilton, Patrick S., and Willis J. Tompkins. "Quantitative investigation of QRS detection rules using the MIT/BIH arrhythmia database." IEEE transactions on biomedical engineering 12 (1986): 1157-1165.

[9] Christov, Ivaylo I. "Real time electrocardiogram QRS detection using combined adaptive threshold." Biomedical engineering online 3.1 (2004): 28. 
[10] Bakardjian, H. "Ventricular beat classifier using fractal number clustering." Medical and Biological Engineering and Computing 30.5 (1992): 495-502.

[11] Zaunseder, Sebastian, Robert Huhle, and Hagen Malberg. "CinC challengeAssessing the usability of ECG by ensemble decision trees." Computing in Cardiology, 2011. IEEE, 2011.

[12] Moody, Benjamin E. "Rule-based methods for ECG quality control." Computing in Cardiology, 2011. IEEE, 2011.

[13] Xia, Henian, et al. "Computer algorithms for evaluating the quality of ECGs in real time." Computing in Cardiology, 2011. IEEE, 2011.

[14] Jekova, Irena, et al. "Recognition of diagnostically useful ECG recordings: Alert for corrupted or interchanged leads." Computing in Cardiology, 2011. IEEE, 2011

[15] Johannesen, Lars. "Assessment of ECG quality on an Android platform." Computing in Cardiology, 2011. IEEE, 2011.

[16] Kalkstein, Nir, et al. "Using machine learning to detect problems in ECG data collection." Computing in Cardiology, 2011. IEEE, 2011.

[17] Tat, Thomas Ho Chee, Chen Xiang, and Lim Eng Thiam. "Physionet challenge 2011: improving the quality of electrocardiography data collected using real time QRS-complex and T-wave detection." Computing in Cardiology, 2011. IEEE, 2011

[18] Starc, Vito. "Could determination of equivalent dipoles from 12 lead ECG help in detection of misplaced electrodes." Computing in Cardiology, 2011. IEEE, 2011.

[19] Vclav Chudek, Luks Zach, Jakub Kuzilek, Jir Spilka, Lenka Lhotsk. "Simple Scoring System for ECG Quality Assessment on Android Platform." Computing in Cardiology, 2011. IEEE, 2011.

[20] Kulek, Jakub, et al. "Data driven approach to ECG signal quality assessment using multistep SVM classification." Computing in Cardiology, 2011. IEEE, 2011.

[21] Dieter Hayn, Bernhard Jammerbund, Gunter Schreier, "ECG Quality Assessment for Patient Empowerment in mHealth Applications," 2011 Computing in Cardiology, Hangzhou, 2011, pp. 353-356. 